\title{
Tissue Engineering: Alternative Option in Managing Large and Massive Tissue Deficits
}

\author{
Ahmad Oryan $^{1 *}$ and Ali Moshiri \\ ${ }^{1}$ Department of Pathology, School of Veterinary Medicine, Shiraz University, Shiraz, Iran \\ ${ }^{2}$ Department of Clinical Sciences, Division of Surgery, School of Veterinary Medicine, Shiraz University, Shiraz, Iran
}

Large and extensive injuries are the most surgical challenges both in humans and animals. There are many different conditions that make a tissue loss including massive traumatic injuries, cancers, and degenerative diseases. Tropical diseases could also be considered as the risk factors of inducing large tissue losses [1-3]. Some physiological conditions such as aging, obesity and repeated pregnancies are also other predisposing items which facilitate injury induction and may result in tissue losses [4]. All these deficits if they are large, their repair is of great concern. Perhaps some of the small soft tissue defects could be repaired by direct suturing, however in such large deficits, the primary surgical repair is of low value [5]

The classical reconstructive surgery is used to repair such large defects and the auto and allografts are gold standard methods. However, transplantation has its own limitations and in some conditions these limitations are significant and depend on the patient inclination, surgeon's experience, equipments, and many other factors [6]. Such limitations include availability of the grafts at the time of surgery, donor site morbidity, cosmetic and biomechanical concerns, transmission viral diseases such as HIV and hepatitis, ethical concerns and cost. Also some of these grafts may not be effective and be resorbed by the body immune response after transplantation [6]. One of the major limitations of the grafts is that they cannot design to be best suited according to the recipient physiologic and functional characteristics so that the healed tissue may not have the same functionality as that of the original tissue [6,7]. Xenografts are another option but with higher limitation than those of the auto and allografts and higher rejection rate could be expected after their application [8].

Tissue engineering is an option and it has a well-documented history [7]. The classical tissue engineered products are only limited to acellularization of the xeno- or allografts [6,9]. In this technology, the cellular structures as the major antigenic source of the graft is rinsed from the tissue but the architectural structure of the graft is kept intact and redesigned in a manner to be best suited to the area of application. However, their application in animal study faced with unclear results $[6,9]$. The newer approaches degrade the biological tissue elements in order to purify the specified molecules such as collagens, with the aiming to redesign a new scaffold for tissue repair. In such technologies, the architectures of the graft could be engineered in order to simulate the normal physiological architecture of the recipient tissue [10]. Based on the type of manufactured molecules and processing technology, different types of bi or tridimensional scaffolds could be produced [9].

Collagens are major biologic molecules that present in almost all body tissues and are biocompatible, biodegradable and safe with the aiming to be applicated in tissue engineering $[6,9,10]$. There are many biomaterials in this field which are either biologic based or synthetic based. Biologic based materials are well tolerated by the body but their processing face with some limitation including cost and availability [6]. The synthetic materials such as polydioxanone, polyglactin and nyolon, some are absorbable, and some are not absorbable but all of them if are used in large scales could initiate significant tissue reactions
$[6,9,10]$. Their merit is related to the high compatibility to engineering processing. They can polymerize in order to produce any size of fibers and can easily arrange in order to produce any types of scaffolds [9]. The more recent products combined the biologic and synthetic compounds to increase the characteristics of the scaffolds such as their architecture organization, biocompatibility and handling $[11,12]$.

Generally there are two types of scaffolds in tissue engineering. Some of them are bidimensional structured and their main purpose is to guide regeneration of the healing tissue in order to establish the continuity of the defect area and their more important role is to reduce adhesions. They are mainly applicable in cutaneous wounds and for tendon and bone healing and for this reason they are wrapped around the defect area $[5,13]$. In such tissue deficits such as tendon, bone, or cartilage defects, with large scale tissue loss, the tridimensional scaffolds are of great value compared to the bidimensional types because they can collaborate in healing response and also they have alignment effect more than bidimensional types $[14,9]$.

In the newer approaches, the tridimensional scaffolds have been combined with the bidimensional ones to increase their efficacy in aligning the regenerated tissue and improve their inhibitory role in adhesions formation to the surrounding areas. Two major technologies have been used to produce these composites including the electro spinning and gelling system $[6,10,11,15]$. The first technology is used to produce the highly aligned scaffolds that are best suited for producing bidimensional scaffolds and the second one is best suited to produce tridimensional scaffolds with the aiming to culture the stem cells in order to produce living tissue engineered grafts $[9,11,15]$. Both of them have limitations and selection of each depends on the application purpose.

There are several healing promotive factors that can be assembled to these scaffolds and delivered at the defect area at specific times to increase the healing rate [16-19]. Although there are many commercially available tissue engineered products however, the basic in vivo and clinical researches are rare; therefore, their efficacy in reconstruction of such tissue deficits is questionable [6]. Most of the present investigations are in vitro experiments and did not consider the host immune reaction in response to tissue engineered graft implantation [9]. It should be stated that the knowledge of tissue healing is important to design well

*Corresponding author: Ahmad Oryan, Department of Pathology, School of Veterinary Medicine, Shiraz University, Shiraz, Iran, E-mail: oryan@shirazu.ac.ir

Received December 12, 2012; Accepted December 12, 2012; Published December 30, 2012

Citation: Oryan A, Moshiri A (2013) Tissue Engineering: Alternative Option in Managing Large and Massive Tissue De icits. Tropical Medicine \& Surgery 1:e101. doi:10.4172/2329-9088.1000e101

Copyright: ( 2013 Oryan A, et al. This is an open-access article distributed under the terms of the Creative Commons Attribution License, which permits unrestricted use, distribution, and reproduction in any medium, provided the original author and source are credited. 
Citation: Oryan A, Moshiri A (2013) Tissue Engineering: Alternative Option in Managing Large and Massive Tissue Deficits. Tropical Medicine \& Surgery 1: e101. doi:10.4172/2329-9088.1000e101

Page 2 of 2

comprehensive detailed study and animal studies are of great value in such circumstance. Although several in vitro researches exist but tissue engineering are still in its elementary phase and could be improved in order to create a new insights with the aiming to initiate a revolution in surgical reconstruction of such large tissue injuries, in near future.

\section{References}

1. Rashid OM, Nagahashi M, Takabe K (2012) Management of massive sof tissue defects: The use of INTEGRA ${ }^{\circledR}$ artificial skin after necrotizing soft tissueinfection of the chest. J Thorac Dis 4: 331-335.

2. Sugiura $H$, Nishida $Y$, Nakashima $H$, Yamada $Y$, Tsukushi $S$, et al. (2012) Evaluation of long-term outcomes of pasteurized autografts in limb salvage surgeries for bone and soft tissue sarcomas. Arch Orthop Trauma Surg 132: 1685-1695.

3. Yoo CM, Kang DH, Hwang SH, Park KB (2012) Reverse latissimus dorsi muscle flap for an extensive soft tissue defect accompanied by infectious spondylitis. J Korean Neurosurg Soc 52: 423-426.

4. Kim J, Lim H, Lee SI, Kim YJ (2012) Thickness of rectus abdominis muscle and abdominal subcutaneous fat tissue in adult women: correlation with age, pregnancy, laparotomy, and body mass index. Arch Plast Surg 39: 528-533.

5. Janfaza M, Martin M, Skinner R (2012) A preliminary comparison study of two noncrosslinked biologic meshes used in complex ventral hernia repairs.World J Surg 36: 1760-1764.

6. Yarlagadda PK, Chandrasekharan M, Shyan JY (2005) Recent advances and current developments in tissue scaffolding. Biomed Mater Eng 15: 159-177

7. Feinberg AW (2012) Engineered tissue grafts: opportunities and challenges in regenerative medicine. Wiley Interdiscip Rev Syst Biol Med 4: 207-220.

8. Veillette CJ, Cunningham KD, Hart DA, Fritzler MJ, Frank CB (1998) Localization and characterization of porcine patellar tendon xenograft antigens in a rabbit model of medial collateral ligament replacement. Transplantation 65: 486-493

9. Chen J, Xu J, Wang A, Zheng M (2009) Scaffolds for tendon and ligament repair: review of the efficacy of commercial products. Expert Rev Med Devices 6: 61-73.

10. Shearn JT, Kinneberg KR, Dyment NA, Galloway MT, Kenter K, et al. (2011) Tendon tissue engineering: progress, challenges, and translation to the clinic. J Musculoskelet Neuronal Interact 11: 163-173.

11. Teng SH, Lee EJ, Wang P, Kim HE (2008) Collagen/hydroxyapatite composite nanofibers by electrospinning. Materials Letters 62: 3055-3058.

12. Appelman TP, Mizrahi J, Elisseeff JH, Seliktar D (2009) The differential effect of scaffold composition and architecture on chondrocyte response to mechanical stimulation. Biomaterials 30: 518-525.

13. Fini M, Torricelli P, Giavaresi G, Rotini R, Castagna A, et al. (2007) In Vitro study comparing two collageneous membranes in view of their clinical application for rotator cuff tendon regeneration. J Orthop Res 25: 98-107.

14. Roeder BA, Kokini K, Sturgis JE, Robinson JP, Voytik-Harbin SL (2002) Tensile mechanical properties of three-dimensional type I collagen extracellular matrices with varied microstructure. J Biomech Eng 124: 214-222.

15. Foltran I, Foresti E, Parma B, Sabatino P, Roveri N (2008) Novel biologically inspired collagen nanofibers reconstituted by electrospinning method. Macromol Symp 269: 111-118.

16. Moshiri A, Oryan A (2011) Structural and Functional Modulation of Early Healing of Full-thickness Superficial Digital Flexor Tendon Rupture in Rabbits by Repeated Subcutaneous Administration of Exogenous Human Recombinant Basic Fibroblast Growth Factor. J Foot Ankle Surg 50: 654-662

17. Oryan A, Moshiri A, Meimandiparizi AH (2011) Effects of sodium-hyaluronate and glucosamine-chondroitin sulfate on remodeling stage of tenotomized superficial digital flexor tendon in rabbits: a clinical, histopathological, ultrastructural, and biomechanical study. Connect Tissue Res 52: 329-339.

18. Oryan A, Moshiri A (2012) Recombinant fibroblast growth protein enhances healing ability of experimentally induced tendon injury in vivo. J Tissue Eng Regen Med.

19. Oryan A, Moshiri A, Raayat AR (2012) Novel Application of Theranekron Enhanced the Structural and Functional Performance of the Tenotomized Tendon in Rabbits. Cells Tissues Organs 196: 442-455. 